\title{
Clinicopathological characteristics of patients with paraproteinemia and renal damage
}

\author{
Xuanli Tang ${ }^{*}$ (D, Feng Wan, Jin Yu, Xiaohong Li, Ruchun Yang and Bin Zhu
}

\begin{abstract}
Background: This study aimed to analyze the clinicopathological characteristics of patients with paraproteinemia and renal damage.

Methods: Ninety-six patients from 2014 to 2018 with paraproteinemia and renal damage were enrolled and the clinical data, renal pathology, treatment and prognosis data were collected.

Results: A total of 96 patients (54 male and 42 female), accounting for $2.7 \%$ of all renal biopsies, were enrolled in this study. Among them, 42 were monoclonal gammopathy of renal significance (MGRS), 21 were renal monotypic immunoglobulin alone (renal monolg), and 19 were monoclonal gammopathy of undetermined significance (MGUS). Individuals with multiple myeloma (MM) accounted for the fewest number of patients ( $n=14)$. In the MGRS group, the main diseases were amyloidosis $(n=25)$ and cryoglobulinemic glomerulonephritis $(n=7)$, while in the MM group, the main diseases were cast nephropathy $(n=9)$ and light chain deposit disease $(n=3)$. In the MGUS group, it was mainly lgA nephropathy (IgAN, $n=10)$ and idiopathic membranous nephropathy $(n=5)$; while in the renal monolg group, most of the cases were IgAN $(n=19)$. Chemotherapy was mainly administered to patients in the MM group, while immunosuppression therapy was mostly administered to patients in the renal monolg group. Most patients with renal monolg exhibited a major response, followed by the patients with MGUS and MGRS, while most of the patients with MM had a partial response but none had a major response. Approximately more than half (57.1\%) of the patients with MM progressed to end-stage renal disease (ESRD), followed by MGRS (33.3\%); however, the mortality rate was low in both the MGRS and MM groups. The survival analysis reviewed that serum creatinine, hemoglobin levels, and the serum $k / \lambda$ ratio were independent risk factors for ESRD in patients with MGRS.
\end{abstract}

Conclusions: The clinicopathological changes in patients with MGRS were between those in patients with MM and MGUS. The treatment for MGRS and MM was more intensive, and the overall mortality rate was low. Both MGUS and renal monolg alone exhibited slighter clinicopathological features than MGRS and MM, and the treatment was focused mostly on primary renal diseases.

Keywords: Paraproteinemia, Monoclonal gammopathy of renal significance, Multiple myeloma, Monoclonal gammopathy of undetermined significance, Renal monotypic immunoglobulin

*Correspondence: tangsunshine1981168@sina.com

Department of Nephrology, Key Laboratory of Kidney Disease Prevention and Control Technology, Hangzhou TCM Hospital Affiliated to Zhejiang

Chinese Medical University, Hangzhou 310007, China

\section{Introduction}

The detection rate of monoclonal immunoglobulin (MIg) has increased due to technological advances and the widespread use of serum and renal paraprotein analysis. Accordingly, renal damage with paraproteinemia is commonly diagnosed among older patients. A paraprotein is 
an MIg or immunoglobulin fragment, which is detected as abnormal serum or urine light and/or heavy chains, or an abnormal light chain ratio. Paraproteins that originate from disorders of the plasma and/or B cells are known as monoclonal gammopathy of undetermined significance (MGUS). MGUS is defined as the presence of a serum monoclonal protein at a concentration of $3.0 \mathrm{~g}$ per deciliter or less, no monoclonal protein or only modest amounts of monoclonal light chains in the urine, the absence of CRAB features (i.e., hypercalcemia, renal insufficiency, anemia, and bone lesions) that are related to the monoclonal protein, and $10 \%$ or fewer monoclonal plasma cells in the bone marrow [1]. According to data from the Mayo Clinic in the United States, the incidence rate of MGUS can be as high as 3\% in Caucasians aged over 50 years $[2,3]$. These patients have no definite lymphocytic disease or do not meet the diagnostic criteria; however, the risk of progressing to lymphocytic hematopoietic malignancies is $1 \%$ per year, and the risk of progression after 25 years is $30 \%$ [4, 5]. MIg is more common in elderly patients aged over 70 years, which might be more possibly combined with immune system disorders, infections, somatic malignancies, or primary kidney diseases $[1,6,7]$. Monoclonal gammopathy of renal significance (MGRS), which refers to monoclonal immunoglobulinemia-associated kidney injury, mostly progresses to renal dysfunction (approximately 72\%), but it can also develop into multiple myeloma (MM; approximately $18 \%)$. Additionally, patients with MGUS progress to more advanced disease at a rate of $1 \%$ per year, and approximately $3 \%$ of patients developed MM out of MGUS [8]. Furthermore, some patients exhibited monotypic immunoglobulin in renal tissue (renal monoIg) by light chain detection, but without MIg in serum. The cause and pathogenesis of renal monoIg is controversial [9-11]. Due to the large differences in kidney damage and prognosis, our study aimed to compare some serological and renal pathological characteristics as well as clinical treatment and prognosis of patients with paraproteinemia and renal damage, especially those with MGRS, MM, MGUS, and renal monoIg.

\section{Materials and methods Patients}

Of the 3502 patients who had renal biopsy in our hospital between 2014 and 2018, 96 patients were enrolled. The presence of MIgs, based on hematology findings, or monotypic immunoglobulins, based on renal biopsy findings, were detected in these patients. Patients were divided into four groups according to different diagnostic criteria. Group 1: MGRS $(n=42)$; a clonal plasma cell or B lymphocyte proliferation causing a renal lesion in the absence of hematologic malignancy or other myeloma-defining events [12]. Group 2: MM $(n=14)$; clonal bone marrow plasma cells $\geq 10 \%$ and the presence of one or more of myeloma-defining events; clonal plasma cells $\geq 60 \%$; serum free light chain ratio of $\geq$ 100; or more than one focal lesion on magnetic resonance imaging [13]. Group 3: MGUS $(n=19)$; < $10 \%$ bone marrow plasma cells, $<3 \mathrm{~g} / \mathrm{dl}$ of monoclonal protein, and no myeloma-defining events [1, 13]. Group 4: the renal monoIg $(n=21)$; the presence of monotypic immunoglobulin in renal tissue without the presence of serum MIg. The indication for kidney biopsy in monoIg group was undetermined hematuria and/or proteinuria. Patients with a history of malignancy treatment, those lost to follow-up, and those with incomplete laboratory and pathological data $(n=3)$ were excluded. The distribution of cases is shown in Fig. 1. This study was approved by the ethical committees of Hangzhou TCM Hospital Affiliated to Zhejiang Chinese Medical University.

\section{Detection of serum monoclonal immunoglobulin}

Serum light chain assays were conducted using an automatic protein analysis system (Siemens, Erlangen, Germany). Immunofixation electrophoresis was performed using an automated Scanning system (Hydrasys 2, Sebia, Lisses, France). All detection tests were based on the manufacturer's instructions.

\section{Detection of renal monotypic immunoglobulin}

Immunofluorescence (IF) of light chains was performed on paraffin-embedded tissues. The tissue was dewaxed, digested with proteinase $\mathrm{K}$ (Dako, Carpinteria, CA), and reacted with polyclonal rabbit anti-human $\kappa$ and $\lambda$ antibodies (1:40 dilution, Dako, Carpinteria, CA, USA). Light chain detection was evaluated and confirmed by

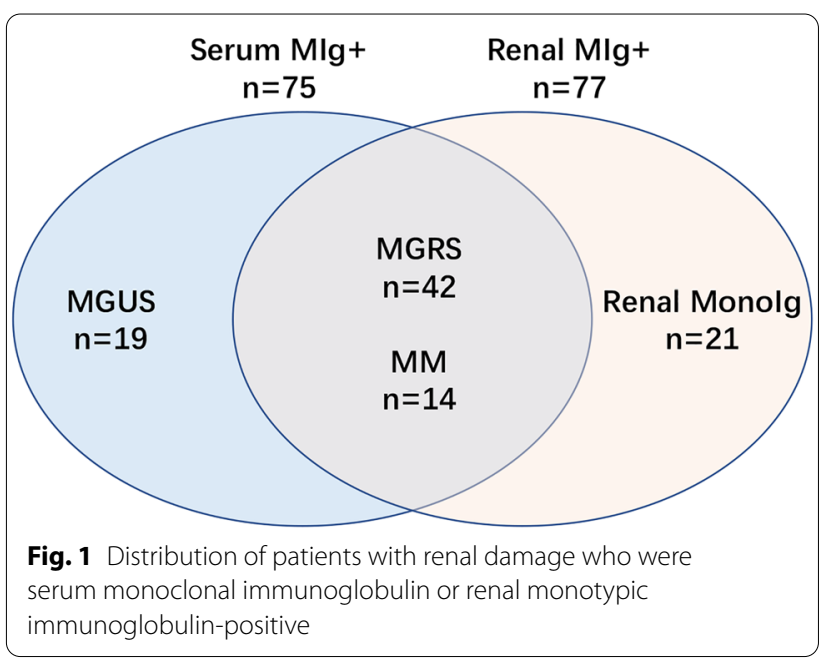


two renal pathologists. Some cases of light chain detection were confirmed using immunoelectron microscopy. Sections measuring $100 \mathrm{~nm}$ were cut using an ultramicrotome and reacted with light chains, and then labeled with immunogold goat anti-rabbit IgG (1:40 dilution, EMS, USA). Sections were then detected under a JOEL1400 electron microscope (JEOL-1400, JEOL Ltd., Peabody, MA, USA).

\section{Clinical and pathological characteristics}

The following demographic and clinicopathological information was collected as followed: age, sex, renal lesion duration, hematuria, 24-h proteinuria, serum albumin, hemoglobin $(\mathrm{Hb})$, serum creatinine $(\mathrm{SCr})$, the estimated glomerular filtration rate (eGFR) (Modification of Diet in Renal Disease formula), serum C3 level, serum/ urine $\kappa / \lambda$ ratio and serum/urine immunofixation electrophoresis (sIFE/uIFE). Pathological features included the diagnosis, the proportion of global and segmental sclerosis, and crescents as well. Interstitial fibrosis/tubular atrophy (IFTA) and interstitial inflammation were estimated according to the proportion of cortex involved and they were divided into three grades $(\leq 25 \%$ as score $1,25-50 \%$ as score 2 , and $\geq 50 \%$ as score 3 ). Podocyte effacement was divided into three grades $(\leq 50 \%$ as score $1,50-75 \%$ as score $2, \geq 75 \%$ as score 3 ). Serum and urine electrophoresis, ANA, ANCA were performed on all patients and anti-PLA2R antibody was performed on patients with idiopathic membranous nephropathy (IMN). Bone marrow aspiration/biopsy was performed on patients with paraproteinemia. The final clinical diagnosis was based on laboratory tests and pathology reports.

\section{Treatment and outcome}

Special treatments included chemotherapy, immunosuppression, and renal replacement (including long-term dialysis and renal transplantation). Chemotherapy regimens consisted mostly of bortezomib combined with dexamethasone, thalidomide combined with dexamethasone, or vincristine and adriamycin combined with dexamethasone. Immunosuppressive drugs consisted mainly of steroids, cyclophosphamide, mycophenolate mofetil, and rituximab. Renal response was defined as major response, partial response, and endpoints [death or end-stage renal disease (ESRD)]. Major response was defined as a reduction in proteinuria to protein excretion $<0.3 \mathrm{~g} / \mathrm{d}$ with stable or improvement of eGFR to $\geq$ $60 \mathrm{~mL} / \mathrm{min} / 1.73 \mathrm{~m}^{2}$; partial response was defined as $\geq$ $50 \%$ decrease (by at least $0.5 \mathrm{~g}$ ) in 24 -h urine protein excretion in the absence of a reduction in eGFR $\geq 25 \%$ or an increase in Scr level $\geq 0.5 \mathrm{mg} / \mathrm{dL}[14,15]$. ESRD was defined as Scr level $>6.0 \mathrm{mg} / \mathrm{dL}$ or being dependent on kidney replacement therapy for more than 6 months
[16]. Patients were followed up in the outpatient clinic or by telephone. A combination of ESRD and all-cause death was defined as the endpoint in the Kaplan-Meier analysis.

\section{Statistical analysis}

Statistical analysis was performed with SPSS 17.0. Normal distribution data were expressed as mean \pm standard deviation (SD). The clinical and pathological characteristics among the groups were compared by $t$ test or analysis of variance (ANOVA) for continuous variables, and by nonparametric tests for discontinuous variables. Categorical variables were expressed as percentages and between-group comparisons were assessed by Chi-square test or Fisher's exact test. Death and renal endpoints were analyzed by univariate Cox regression followed by multivariate Cox regression. Predictive models were created using clinical data and pathological data. Univariate variables included the following variables: age, gender, lesion duration, hematuria, proteinuria, albumin, Hb, Scr, eGFR, low serum C3 level, abnormal serum and urine $\kappa / \lambda$ ratio, abnormal sIFE and uIFE, presence of renal monotypic $\kappa$, glomerular global and segmental sclerosis, crescents, IFTA, interstitial inflammation, and podocyte effacement. Multivariate variables included $\mathrm{Hb}$, Scr, eGFR, abnormal serum and urine $\kappa / \lambda$ ratio, and glomerular global and segmental sclerosis. The KaplanMeier analysis was used to compare the survival rates. Results were expressed as hazard ratio with 95\% confidence intervals. All $P$ values were two-sided, and statistical significance was set at $P<0.05$.

\section{Results}

\section{Demographic and clinicopathological data of patients with MGRS, MM, MGUS, and renal monolg}

A total of 96 patients (54 male and 42 female), were enrolled in this study, which accounted for $2.7 \%$ of all renal biopsies, performed in our hospital during the study period. The mean age of the patients was $53.3 \pm$ 12.8 years, and the participants were divided into four groups. MGRS accounted for $43.7 \%(n=42)$ of all cases, followed by the renal monoIg and MGUS groups (21.9\% and $19.8 \%$, respectively). MM accounted for the fewest number of cases (14.6\%). In the MGRS group, amyloidosis was the main disease, affecting $59.5 \%(n=25)$ of cases. In the MM group, cast nephropathy was the main disease $(n=9)$, and three of these cases had light chain deposit disease (LCDD) combined with MM (Fig. 2). In the MGUS group, patients were mainly diagnosed with primary or secondary IgA nephropathy $(\operatorname{IgAN})(n=$ 8 and $n=2$, respectively), followed by IMN $(n=5)$, mesangial proliferation nephritis $(n=2)$, acute tubular injury $(n=1)$, and malignant hypertension $(n=1)$. 

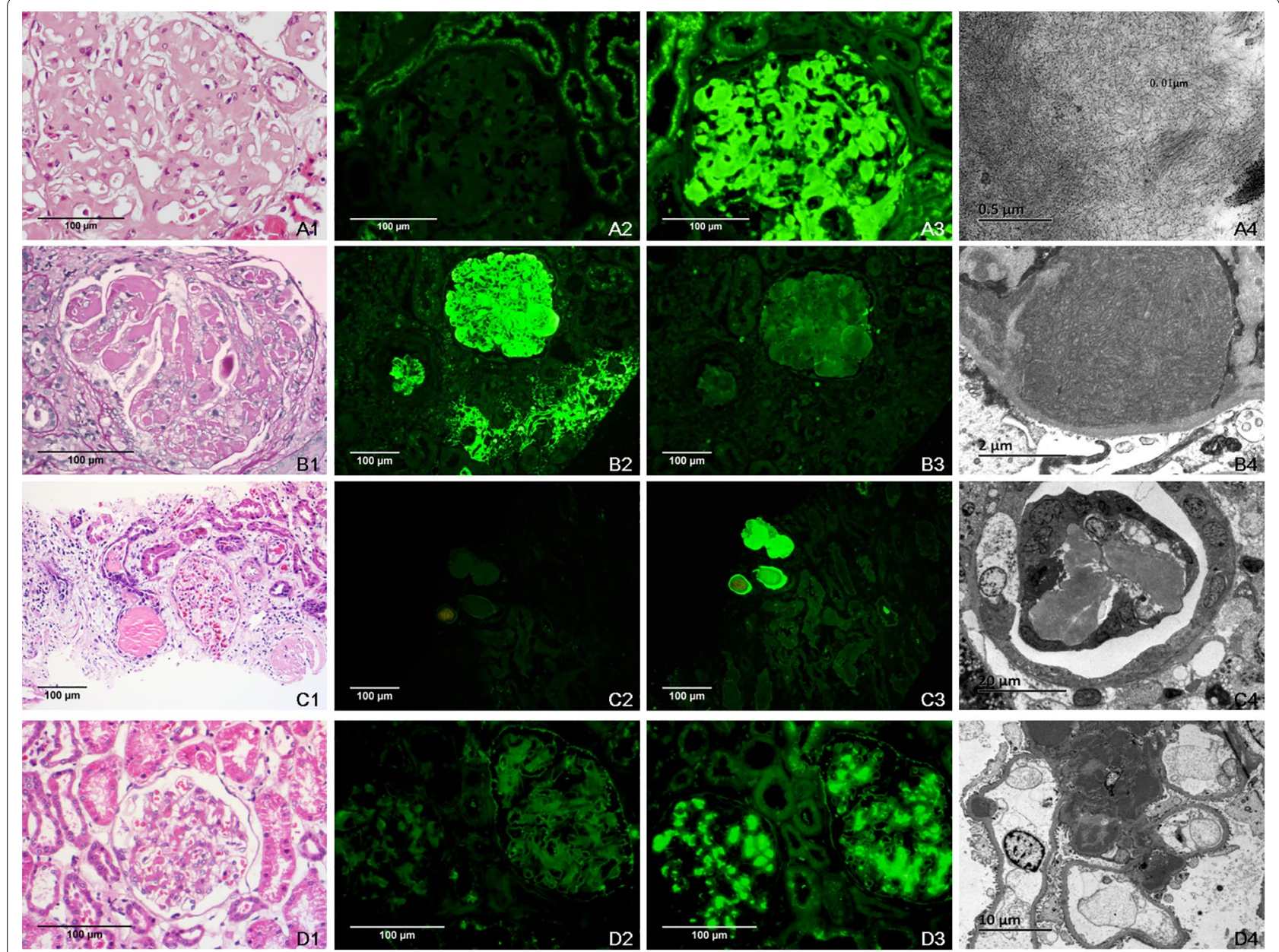

Fig. 2 Renal pathological characteristics in some patients with serum monoclone or renal monolg positivity. A-D Representative cases of amyloidosis, cryoglobulinemic glomerulonephritis, cast nephropathy and light chain restricted lgA nephropathy. A 1 The glomerulus exhibited expansion of the mesangium and thickening of capillary walls by amorphous material $(H E, \times 400) ; \mathbf{A} 2$ negative $k$ light chain and $\mathbf{A} 3$ positive $\lambda$ light chain (IF, $\times$ 400); A4 8-12 nm nonbranching fibrils in the subepithelial and mesangial areas (EM, $\times 40,000)$; $\mathbf{B} 1$ the glomerulus exhibited cryoglobulin pseudothrombi in the glomerular capillary lumens (HE, $\times 400) ; \mathbf{B} 2$ positive $k$ light chain and $\mathbf{B} 3$ negative $\lambda$ light chain (IF, $\times 200) ; \mathbf{B} 4$ large and curved fibrils seen in subendothelial deposits (EM, $\times 10,000) ; \mathbf{C} 1$ a large eosinophilic and fractured cast with interstitial inflammation $(H E, \times 200) ; C 2$ negative $k$ light chain and $\mathbf{C} 3$ positive $\lambda$ light chain $(I F, \times 200) ; C 4$ fractured cast with multinucleated giant cell reaction (EM, $\times$ 1000); D1 the glomerulus showed mesangial proliferation (HE, $\times$ 400); D2 negative $k$ light chain and D3 positive $\lambda$ light chain (IF, $\times 400) ; \mathbf{D} 4$ mesangial expansion with amorphous dense deposits $(E M, \times 2000)$

In the renal monoIg group, most of the cases had IgAN $(n=19)$. The remaining two cases were lupus nephritis (LN) and hepatitis B-related nephritis. A comparison of the clinicopathological characteristics is shown in Table 1. Among those four groups, patients with MGRS were found to exhibit lower levels of albumin and worse podocyte effacement compared to the other groups $(P<0.001)$. Additionally, the sIFE-positive detection rate was lower in the MGRS group than in the MM and MGUS groups $(P<0.001)$. Patients with MM exhibited a much lower levels of hemoglobin, yet less hematuria $(P<0.001$ and $P=0.021$, respectively), the highest
SCr levels and the lowest eGFR than some of the other groups $(P=0.002$ and $P=0.054$, respectively). Additionally, patients with $\mathrm{MM}$ had a higher abnormal $\kappa / \lambda$ ratio in both the serum and urine compared to the other groups $(P=0.024)$, and their interstitial fibrosis and inflammation were worse $(P=0.029)$. The MGUS group exhibited the highest levels of hemoglobin and the lowest levels of serum creatinine $(P<0.001$ and $P=0.002$, respectively). No renal monoclonal light chain deposits were detected. Renal monoIg patients were the youngest, and had more hematuria and less proteinuria than some of the other groups $(P<0.001, P=0.021$, and $P=$ 
Table 1 Clinicopathological characteristics of patients with paraproteinemia and renal damage

\begin{tabular}{|c|c|c|c|c|c|}
\hline & MGRS $(n=42)$ & $\mathrm{MM}(n=14)$ & MGUS $(n=19)$ & Renal monolg $(n=21)$ & $P$ value \\
\hline Male $[n(\%)]$ & $25(59.5)$ & $9(64.3)$ & $12(63.2)$ & $8(38.1)$ & 0.292 \\
\hline Age (year) & $57.0 \pm 10.6$ & $57.0 \pm 7.0$ & $53.2 \pm 11.5$ & $43.3 \pm 15.9^{c, e, f}$ & 0.000 \\
\hline $\begin{array}{l}\text { Renal disease duration before renal } \\
\text { biopsy (months) }\end{array}$ & $11.0 \pm 11.1$ & $6.3 \pm 8.1$ & $21.1 \pm 44.1$ & $26.8 \pm 57.5$ & 0.210 \\
\hline Hematuria [n (\%)] & $12(27.9)$ & $2(15.4)^{d}$ & $10(52.6)$ & $12(57.1)^{c, e}$ & 0.021 \\
\hline Proteinuria (g/24 h) & $3.9 \pm 2.7$ & $3.3 \pm 2.6$ & $2.8 \pm 2.6$ & $1.8 \pm 2.3^{c}$ & 0.026 \\
\hline Albumin (g/L) & $27.7 \pm 7.0^{a, b, c}$ & $36.6 \pm 6.8$ & $32.7 \pm 7.4$ & $35.3 \pm 7.1$ & 0.000 \\
\hline Hemoglobin (g/L) & $113.6 \pm 21.3$ & $99.9 \pm 16.7^{\mathrm{a}, \mathrm{d}, \mathrm{e}}$ & $131.2 \pm 16.3^{b}$ & $122.9 \pm 19.6$ & 0.000 \\
\hline $\mathrm{SCr}(\mu \mathrm{mol} / \mathrm{L})$ & $160.2 \pm 205.8$ & $359.1 \pm 385.4^{\mathrm{a}, \mathrm{d}, \mathrm{e}}$ & $102.6 \pm 103.5$ & $104.1 \pm 60.2$ & 0.002 \\
\hline $\mathrm{eGFR}\left(\mathrm{mL} / \mathrm{min} / 1.73 \mathrm{~m}^{2}\right)$ & $68.9 \pm 39.7$ & $45.3 \pm 43.6^{\mathrm{a}, \mathrm{d}, \mathrm{e}}$ & $77.7 \pm 25.4$ & $75.3 \pm 34.9$ & 0.054 \\
\hline Low serum C3 [n (\%)] & $15(35.7)$ & $3(21.4)$ & $1(5.3)^{b, f}$ & $7(33.3)$ & 0.078 \\
\hline Abnornal serum $k / \lambda$ ratio $[n(\%)]$ & $19(45.2)$ & $11(78.6)^{a, d}$ & $8(42.1)$ & $0(0)^{c, e, f}$ & 0.000 \\
\hline Abnormal urine $\kappa / \lambda$ ratio $[n(\%)]$ & $17(42.5)$ & $13(92.9)^{\mathrm{a}, \mathrm{d}}$ & $5(29.4)$ & $0(0)^{c, e, f}$ & 0.000 \\
\hline Abnormal sIFE [n (\%)] & $25(61.0)^{a, b}$ & $13(92.9)$ & $19(100)$ & $0(0)^{c, e, f}$ & 0.000 \\
\hline Abnormal ulFE $[n(\%)]$ & $23(76.7)$ & $12(85.7)$ & $8(53.3)$ & $0(0)^{c, e, f}$ & 0.002 \\
\hline K slFE, [n (\%)] & $9(37.5)$ & $7(53.8)$ & $11(57.9)$ & $0(0)$ & 0.371 \\
\hline k ulFE, $[n(\%)]$ & $5(22.7)$ & $6(50.0)$ & $4(50.0)$ & $0(0)$ & 0.183 \\
\hline Renal monotypic $\kappa$ [n (\%)] & $16(40.0)$ & $7(53.8)$ & $0(0)^{b, d}$ & $0(0)^{c, e, f}$ & 0.000 \\
\hline Global glomerulosclerosis (\%) & $16.7 \pm 18.0$ & $17.9 \pm 24.3$ & $13.3 \pm 10.1$ & $15.1 \pm 16.0$ & 0.863 \\
\hline Segmental glomerulosclerosis (\%) & $0.8 \pm 3.7$ & $1.1 \pm 1.9$ & $1.2 \pm 3.0$ & $1.5 \pm 2.9$ & 0.223 \\
\hline Crescents (\%) & $4.1 \pm 14.4$ & $2.2 \pm 3.8^{e}$ & $4.1 \pm 7.6$ & $13.3 \pm 2.9$ & 0.024 \\
\hline IFTA & $1.29 \pm 0.84$ & $2.0 \pm 1.18^{\mathrm{a}, \mathrm{d}}$ & $1.05 \pm 0.71$ & $1.29 \pm 0.90$ & 0.029 \\
\hline Interstitial inflammation & $1.20 \pm 0.90$ & $2.00 \pm 1.17^{\mathrm{a}, \mathrm{d}}$ & $1.11 \pm 0.81$ & $1.52 \pm 1.03$ & 0.000 \\
\hline
\end{tabular}

MGRS monoclonal gammopathy of renal significance; MM multiple myeloma; MGUS monoclonal gammopathy of undetermined significance; MIg monoclonal immunoglobulin; monolg monotypic immunoglobulin; SCr serum creatinine; GFR the estimated glomerular filtration rate; sIFE serum immunofixation electrophoresis; uIFE urine immunofixation electrophoresis; IFTA interstitial fibrosis/tubular atrophy

a $P<0.05$, MGRS vs MM group

${ }^{\mathrm{b}} P<0.05$, MGRS vs MGUS group

c $P<0.05$, MGRS vs renal monolg group

$\mathrm{d} P<0.05$, MM vs MGUS group

e $P<0.05$, MM vs renal monolg group

${ }^{f} P<0.05$, MGUS vs renal monolg group

0.026 , respectively). No hematological paraproteins were detected according to the abnormal light chain ratio or IFE.

\section{Clinicopathological features of patients with MGRS}

Most patients with MGRS had amyloidosis $(\mathrm{n}=25)$, followed by cryoglobulinemic glomerulonephritis (Cryo-GN, $n=7$ ), proliferative glomerulonephritis with monoclonal IgG deposits (PGNMID, $n=5$ ), and monoclonal immunoglobulin deposition disease (MIDD, $n=3$ ). The clinicopathological data of these patients are summarized in Table 2. Patients with C3 glomerulonephritis (C3GN) and immunotactoid glomerulonephritis (ITG) were excluded due to the limited number of cases (only one case each). Patients with amyloidosis had less hematuria than those with CryoGN or PGNMID $(P=0.001)$, and their Hb level was the highest $(P<0.001)$. Patients with Cryo-GN had the highest level of proteinuria while those with MIDD had the highest albumin levels $(P=0.002$ and $P=0.017$, respectively). Patients with MIDD had the highest $\mathrm{SCr}$ levels and the lowest eGFR $(P=0.002$ and $P=0.001$, respectively), followed by those with Cryo-GN. More patients with PGNMID had low serum C3 levels than those with amyloidosis. The abnormal $\kappa / \lambda$ ratio and IFE were similar among the groups. Patients with amyloidosis exhibited a lower proportion of serum and renal monoclonal $\kappa$ chains than those with Cryo-GN and PGNMID $(P=0.031$ and $P=0.003$, respectively). Patients with MIDD exhibited worse chronic pathological features, including interstitial fibrosis and inflammation $(P<0.001)$. 
Table 2 Clinicopathological characteristics of patients in MGRS group

\begin{tabular}{|c|c|c|c|c|c|}
\hline & Amyloidosis ( $n=25$ ) & Cryo-GN $(n=7)$ & $\operatorname{MIDD}(n=3)$ & PGNMID $(n=5)$ & $P$ value \\
\hline Male $[n(\%)]$ & $17(68.0)$ & $4(57.1)$ & $2(66.7)$ & $2(38.1)$ & 0.336 \\
\hline Age (year) & $58.0 \pm 7.7$ & $53.3 \pm 16.4$ & $69.7 \pm 8.4$ & $56.4 \pm 7.5$ & 0.209 \\
\hline $\begin{array}{l}\text { Renal disease duration before renal } \\
\text { biopsy (months) }\end{array}$ & $10.0 \pm 10.8$ & $7.8 \pm 7.0$ & $22.0 \pm 22.7$ & $13.7 \pm 10.3$ & 0.289 \\
\hline Hematuria $[n(\%)]$ & $2(23)^{a, c}$ & $6(85.7)$ & $1(33.3)$ & $3(60.0)$ & 0.001 \\
\hline Proteinuria (g/24 h) & $3.8 \pm 2.3$ & $7.0 \pm 2.9^{\mathrm{a}, \mathrm{d}, \mathrm{e}}$ & $2.0 \pm 1.6$ & $2.1 \pm 1.5$ & 0.002 \\
\hline Albumin (g/L) & $25.8 \pm 5.8$ & $25.4 \pm 3.9$ & $36.6 \pm 1.7^{b, d}$ & $31.1 \pm 9.7$ & 0.017 \\
\hline Hemoglobin (g/L) & $123.4 \pm 16.5^{a, b, c}$ & $91.1 \pm 12.5$ & $93.0 \pm 28.2$ & $104.6 \pm 16.5$ & 0.000 \\
\hline $\mathrm{SCr}(\mu \mathrm{mol} / \mathrm{L})$ & $134.2 \pm 216.8$ & $243.3 \pm 197.3$ & $386.3 \pm 221.0^{b, f}$ & $78.6 \pm 33.1^{\mathrm{e}}$ & 0.002 \\
\hline $\mathrm{eGFR}\left(\mathrm{mL} / \mathrm{min} / 1.73 \mathrm{~m}^{2}\right)$ & $81.7 \pm 37.6$ & $29.0 \pm 17.1^{\mathrm{a}, \mathrm{e}}$ & $18.4 \pm 10.7^{b, f}$ & $80.1 \pm 30.4$ & 0.001 \\
\hline Low serum C3 [n (\%)] & $4(16.0)^{c}$ & $3(42.9)$ & $2(66.7)$ & $5(100)$ & 0.089 \\
\hline Abnormal serum $k / \lambda$ ratio $[n(\%)]$ & $13(52.0)$ & $3(42.9)$ & $2(66.7)$ & $0(0)$ & 0.269 \\
\hline Abnormal urine $k / \lambda$ ratio $[n(\%)]$ & $14(60.9)$ & $1(14.3)$ & $2(66.7)$ & $0(0)$ & 0.131 \\
\hline Abnormal sIFE [n (\%)] & $16(66.7)$ & $5(71.4)$ & $2(66.7)$ & $1(20)$ & 0.237 \\
\hline Abnormal ulFE [n (\%)] & $17(77.3)$ & $3(75)$ & $2(100)$ & $1(50)$ & 0.702 \\
\hline K (sIFE, [n (\%)]) & $3(20.0)^{a}$ & $4(80)$ & $1(50)$ & $0(0)$ & 0.031 \\
\hline K (ulFE, $[n(\%)])$ & $3(18.8)$ & $1(33.3)$ & $1(50.0)$ & $0(0)$ & 0.687 \\
\hline Renal monotypic $k$ [n (\%)] & $4(16.0)^{c}$ & $5(71.4)$ & $2(66.7)$ & $4(80)$ & 0.003 \\
\hline Global glomerulosclerosis (\%) & $14.4 \pm 16.4$ & $17.4 \pm 22.8$ & $42.3 \pm 13.7^{\mathrm{b}, \mathrm{d}, \mathrm{f}}$ & $12.6 \pm 13.8$ & 0.081 \\
\hline Segmental glomerulosclerosis (\%) & 0 & $3.1 \pm 8.3$ & 0 & $2 \pm 4.5$ & 0.236 \\
\hline Crescents (\%) & 0 & $18.0 \pm 32.2^{\mathrm{a}}$ & 0 & $8.2 \pm 6.8$ & 0.319 \\
\hline IFTA & $1.04 \pm 0.69^{\mathrm{a}}$ & $1.86 \pm 0.69^{e}$ & $3.0 \pm 0.0^{b, d, f}$ & $1.0 \pm 0.0$ & 0.000 \\
\hline Interstitial inflammation & $0.79 \pm 0.66^{\mathrm{a}, \mathrm{b}}$ & $2.14 \pm 0.69$ & $2.33 \pm 0.58$ & $1.40 \pm 0.89$ & 0.000 \\
\hline Podocyte effacement (\%) & $2.6 \pm 0.6$ & $2.6 \pm 0.8$ & $2.3 \pm 0.6$ & $2.4 \pm 0.9$ & 0.883 \\
\hline
\end{tabular}

MGRS monoclonal gammopathy of renal significance; MM multiple myeloma; MGUS monoclonal gammopathy of undetermined significance; MIg monoclonal immunoglobulin; monolg monotypic immunoglobulin; SCr serum creatinine; eGFR the estimated glomerular filtration rate; sIFE serum immunofixation electrophoresis; uIFE urine immunofixation electrophoresis; IFTA interstitial fibrosis/tubular atrophy

a $P<0.05$, Amyloid vs Cryo group

${ }^{\mathrm{b}} P<0.05$, Amyloid vs MIDD group

${ }^{c} P<0.05$, Amyloid vs PGNMID group

${ }^{\mathrm{d}} P<0.05$, Cryo vs MIDD group

e $P<0.05$, Cryo vs PGNMID group

${ }^{f} P<0.05$, MIDD vs PGNMID group

\section{Treatment and survival analysis of patients with MGRS, MM, MGUS and renal monolg}

Most of the patients in the four groups underwent special treatment, including chemotherapy and immunosuppression (Table 3). Chemotherapy was administered mostly to patients with MM, and it was administered significantly more to patients with MM than to those with MGRS $(P<$ 0.001). Immunosuppression therapy was administered mostly to patients in the renal monoIg group, followed by those in the MGUS and MGRS groups, each of which were significantly different $(P<0.001)$. Long-term dialysis or renal transplantation was only applied in patients with MGRS and MM who had ESRD. The average followup period was $44.8 \pm 16.9$ months. The renal monoIg group had the highest proportion of patients with major response, followed by the MGUS and MGRS groups, each of which were significantly different $(P<0.001)$.
The MM group had a higher proportion of patients with partial response, but none of the patients showed a major response. More than half of the patients with MM (57.1\%) reached ESRD, followed by those with MGRS (33.3\%), although both the MGRS and MM groups had a low mortality rate without significant difference $(16.7 \%$ vs $21.4 \%, P>0.05)$. The difference in survival time between these patients was also not significant (16.7\% vs. $21.4 \%)$. Patients with MGUS demonstrated a very low rate of ESRD progression, and none of the patients with MGUS or renal monoIg died during the observational time.

By Kaplan-Meier analysis, renal survival was significantly better in the MGUS and renal monoIg groups than in the MGRS and MM groups $(P<0.05)$, although it had no significant difference between the MGRS and MM groups $(P>0.05$; Fig. $3 a)$. The overall survival was significantly better in the renal monoIg group than in either 
Table 3 Treatment and survival analysis of patients with paraproteinemia and renal damage

\begin{tabular}{|c|c|c|c|c|c|}
\hline & MGRS $(n=42)$ & $\mathrm{MM}(n=14)$ & MGUS $(n=19)$ & Renal monolg $(n=21)$ & $P$ value \\
\hline Special treatment [n (\%)] & $36(85.7)$ & $13(92.9)$ & $13(68.4)$ & $18(85.7)$ & 0.239 \\
\hline Chemotherapy [n (\%)] & $19(45.2)^{a, b, c}$ & $12(85.7)^{\mathrm{d}, \mathrm{e}}$ & $0(0)$ & $0(0)$ & 0.000 \\
\hline Immunosuppression [n (\%)] & $12(28.6)^{a, b, c}$ & $0(0)^{d, e}$ & $12(63.2)$ & $18(85.7)$ & 0.000 \\
\hline Follow-up time (months) & $47.6 \pm 19.2$ & $50.9 \pm 18.4$ & $38.9 \pm 14.7$ & $40.4 \pm 10.0$ & 0.080 \\
\hline Major response [n (\%)] & $10(23.8)^{a, b, c}$ & $0(0)^{d, e}$ & $11(57.9)^{f}$ & $18(85.7)$ & 0.000 \\
\hline Partial response $[n(\%)]$ & $16(38.1)$ & $6(42.9)$ & $5(26.3)$ & $3(14.3)$ & 0.185 \\
\hline $\operatorname{ESRD}[n(\%)]$ & $14(33.3)^{b}$ & $8(57.1)^{\mathrm{d}, \mathrm{e}}$ & $1(5.3)$ & $0(0)$ & 0.025 \\
\hline Baseline eGFR (mL/min) & $50.0(14.7,89.0)$ & $15.6(5.8,34.7)$ & 22 & - & 0.732 \\
\hline Renal survival time (months) & $8.8 \pm 10.0$ & $9 \pm 16.6$ & 2 & - & 0.507 \\
\hline Death $[n(\%)]$ & $7(16.7)^{b, c}$ & $3(21.4)^{\mathrm{d}, \mathrm{e}}$ & $0(0)$ & $0(0)$ & 0.014 \\
\hline Survival time (months) & $9.4 \pm 8.4$ & $8.0 \pm 6.1$ & - & - & 0.651 \\
\hline
\end{tabular}

Baseline eGFR and renal survival time were of ESRD patients, while survival time was of died patients

Special treatments included chemotherapy, immunosuppression, and renal replacement (including long-term dialysis and renal transplantation). Chemotherapy regimens consisted mostly of bortezomib combined with dexamethasone (BD regimen), thalidomide combined with dexamethasone (TD regimen), or vincristine and adriamycin combined with dexamethasone (VAD regimen). Immunosuppressive drugs consisted mainly of steroids, cyclophosphamide, mycophenolate mofetil, and rituximab

ESRD end-stage renal disease, IS immunosuppression

a $P<0.05$, MGRS vs MM group

${ }^{\mathrm{b}} P<0.05$, MGRS vs MGUS group

${ }^{c} P<0.05$, MGRS vs renal monolg group

${ }^{\mathrm{d}} P<0.05, \mathrm{MM}$ vs MGUS group

e $P<0.05, M M$ vs renal monolg group

${ }^{f} \mathrm{P}<0.05$, MGUS vs renal monolg group

the MGRS or the MM group $(P=0.05$ and $P=0.03$, respectively), and it was also better in the MGUS group than the MM group $(P=0.04$; Fig. $3 \mathrm{~b})$. Among the patients with MGRS, those with MIDD had much poorer renal survival compared with those in the other groups $(P<0.05)$, although the overall survival was not significantly different between them (Fig. 4).

Survival analysis identified eGFR, abnormal urine $\kappa / \lambda$ ratio, and the presence of renal monoclonal light chains as risk factors, but they were not independent risk factors for mortality in the MGRS group. However, SCr, $\mathrm{Hb}$, and the serum $\kappa / \lambda$ ratio were identified as independent risk factors for ESRD in the MGRS group (Table 4). There were no risk factors for death in the MM group, and although SCr, eGFR, and interstitial fibrosis were risk factors, they were not independent for ESRD.

\section{Discussion}

There is a higher prevalence of both MGUS and chronic kidney disease (CKD) in older patients. The same patient could therefore have unrelated hematological conditions and CKD. As such, distinguishing the conditions is essential because of their different clinical characteristics and associated therapies [17]. MGRS can only be confirmed by renal biopsy, which has been widely used to distinguish it from different types of renal injury.
It has recently been reported that clinically suspected MGUS had $40 \%$ of patients progressing to MGRS, while another study showed only $10 \%$ years ago $[6,18]$. In our study, $56 \%, 25.3 \%$ and $18.7 \%$ of patients with monoclonal gammopathy who underwent renal biopsy had MGRS, MGUS with unrelated CKD, and MM. Two (4.5\%) patients who were initially diagnosed with MGRS progressed and were reclassified as having $M M$ after a second bone marrow biopsy, although the progression rate was previously reported to be $18 \%$ [8]. Amyloidosis was diagnosed in $59.5 \%$ of patients with MGRS, which is consistent with the findings of previous studies $[6,19,20]$ and may explain the lower albumin level and worse podocyte effacement in MGRS group than the other groups. Among the patients with MM, 64.3\% had cast nephropathy, which is consistent with a previous study [21]. This likely explains the lower eGFR and $\mathrm{Hb}$ level, higher SCr level and paraprotein detection rate, and the worse chronic pathological lesions in the MM groups than in the other groups. In the present study, patients with MGUS were mainly diagnosed with primary IgAN, followed by IMN, which may explain the lower serum C3 levels and greater podocyte effacement in the MGUS group than in the other groups. Additionally, renal monoIg deposit diseases without hematological monoclonal gammopathy are poorly understood. Our 


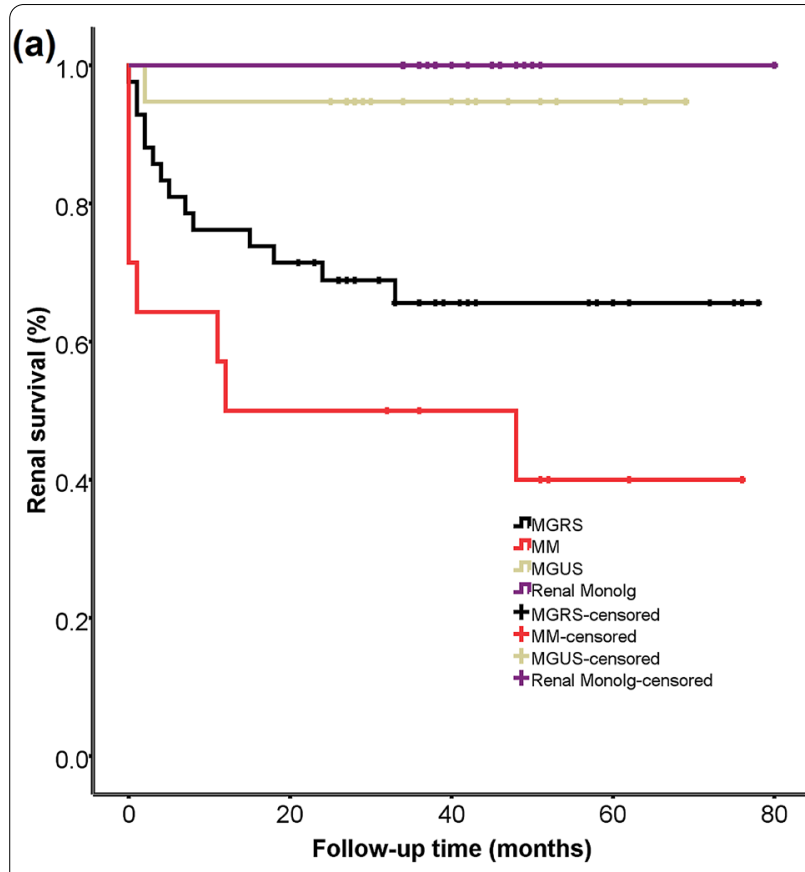

(b)

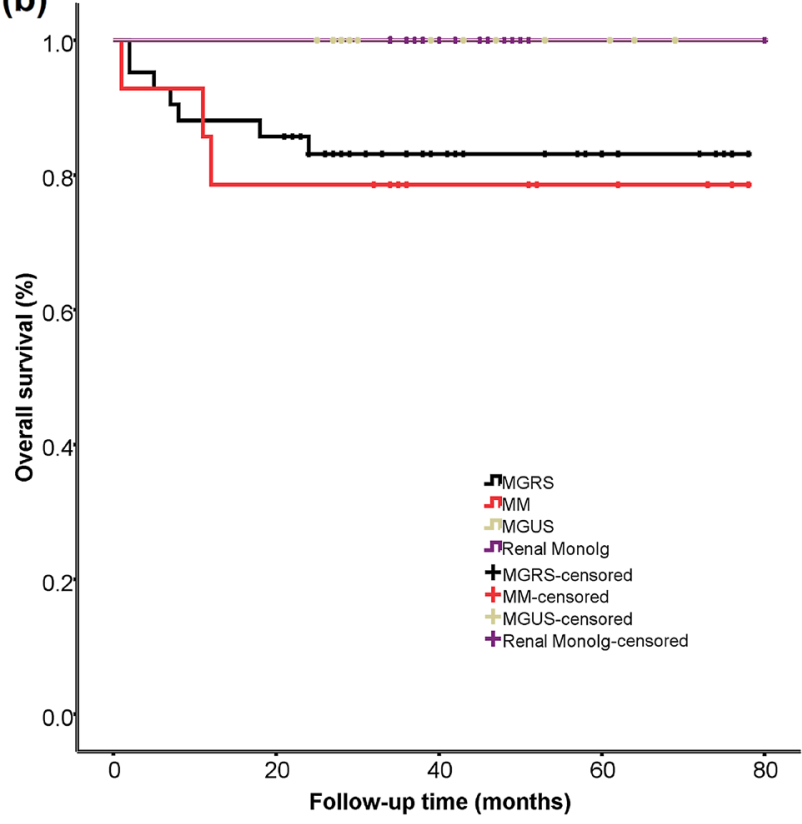

Fig. 3 Comparison of renal survival $\mathbf{a}$ and overall survival $\mathbf{b}$ in patients with MGRS, MM, MGUS, and renal monolg

study demonstrated that most (90.5\%) of these patients had IgAN with $\lambda$ restrictions, and they were much younger than patients with MGRS, MGUS, and MM. They had less proteinuria and lower Scr levels, and had more hematuria, but no paraprotein was detected in their serum or urine samples during follow-up. These data indicate mild pathogenicity in these patients compared

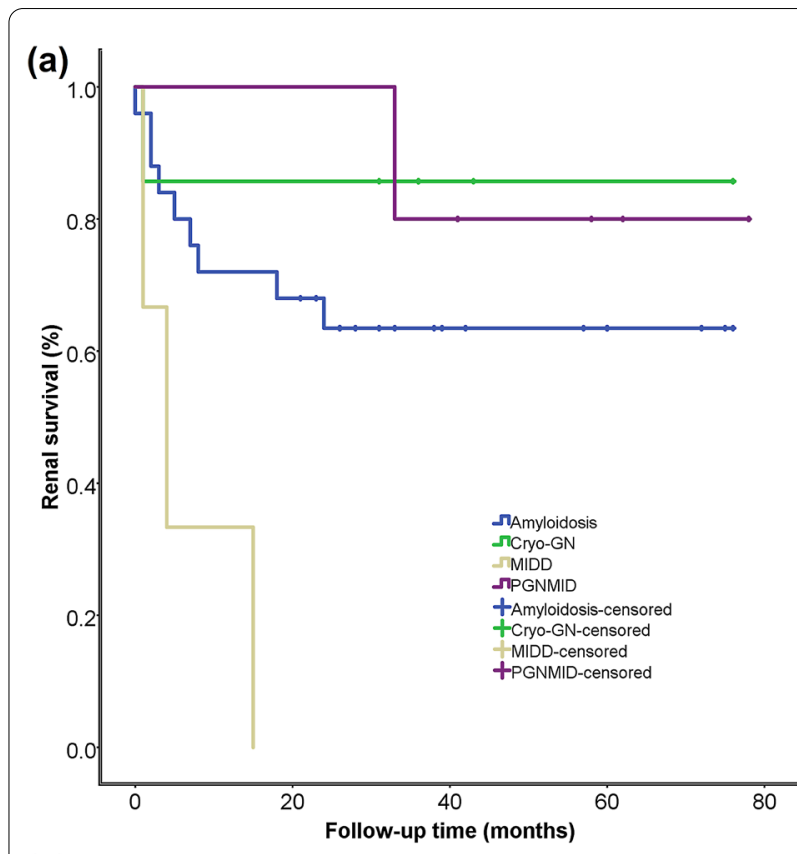

(b)

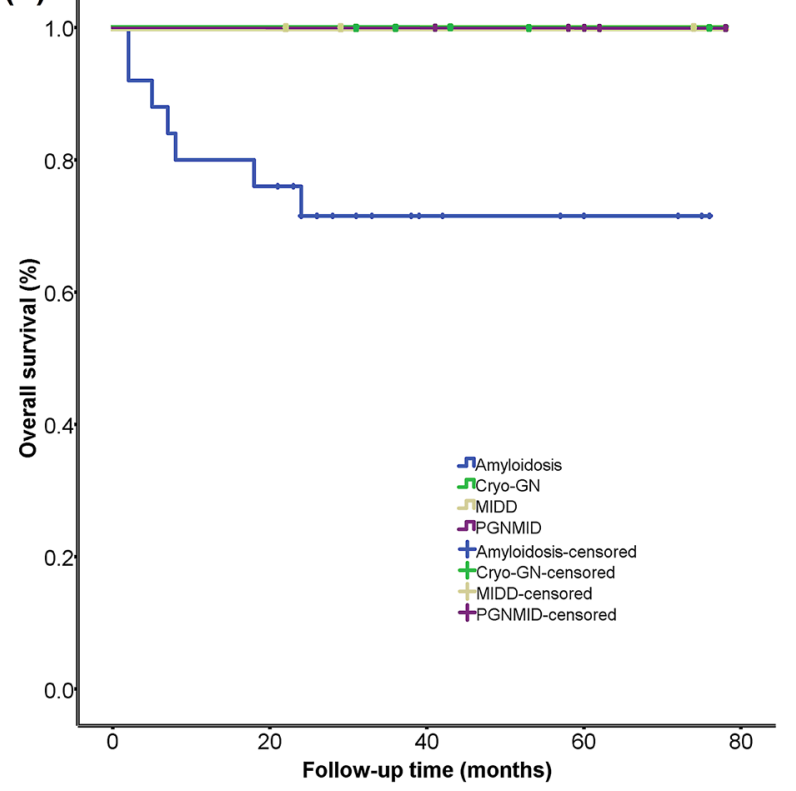

Fig. 4 Comparison of renal survival $\mathbf{a}$ and overall survival $\mathbf{b}$ in patients with MGRS who have amyloidosis, Cryo-GN, MIDD, and PGNMID

to paraprotein-related kidney injuries. Boumediene et al. [22] compared monoclonal IgAN with primary IgAN and found that $\lambda$ restricted IgA1 had hyposialylation, but no hypogalactosylation, which provides low isoelectric point (pI) and high affinity of mesangial region, causing selective deposition. However, Vignon et al. [10] reported that monotypic IgA deposits in such as heavy chain restricted 
Table 4 Independent risk factors for ESRD of MGRS

\begin{tabular}{|c|c|c|c|c|}
\hline & MGRS & & & \\
\hline & $H R$ & $95 \% \mathrm{Cl}$ lower & $95 \% \mathrm{Cl}$ upper & $P$ \\
\hline Scr & 1.004 & 1.002 & 1.006 & 0.000 \\
\hline $\mathrm{Hb}$ & 1.036 & 1.000 & 1.073 & 0.049 \\
\hline Serum $k / \lambda$ ratio & 3.550 & 1.176 & 10.713 & 0.025 \\
\hline
\end{tabular}

Univariate variables included the following variables: age, gender, lesion duration, hematuria, proteinuria, albumin, $\mathrm{Hb}$, Scr, eGFR, low serum C3 level, abnormal serum and urine $\kappa / \lambda$ ratio, abnormal sIFE and UIFE, presence of renal monotypic $k$, glomerular global and segmental sclerosis, crescents, IFTA, interstitial inflammation, and podocyte effacement. Multivariate variables included $\mathrm{Hb}, \mathrm{Scr}$, eGFR, abnormal serum and urine $\kappa / \lambda$ ratio, and glomerular global and segmental sclerosis

IgAN and IgA-PGNMID exhibited distinct clinical, histological, and pathophysiological features from polytypic IgA deposits, with a $35.7 \%$ paraprotein detection rate according to IFE. Therefore, long-term follow-up is essential for patients with renal monoIg. Furthermore, there were two patients with LN and hepatitis B-related glomerulonephritis (HBV-GN), which showed $\lambda$ restriction in renal tissue. We hypothesized that this was due to the selectivity difference of two light chains, according to the similar treatment and prognosis of LN or HBV-GN, and no MIg was detected during follow-up.

MGRS is complex and heterogenous, and manifests in diverse patterns of renal injury [13, 23, 24]. Consensus regarding the evaluation of MGRS was reached and released in 2019 [12]. In the present study, amyloidosis was the most common disease in patients with MGRS, followed by Cryo-GN, PGNMID, and MIDD. These findings were consistent with those of a previous study [20]. The incidence of ITG and C3GN is low in patients with MGRS [25, 26], and in this study we encountered only one case of each. Patients with amyloidosis had less hematuria, higher $\mathrm{Hb}$ levels, lesser proportion of abnormal $\kappa$ chains, and milder IFTA and inflammation due to its fibril features without activation of the complement pathway [27]. Patients with Cryo-GN exhibited more proteinuria, lower eGFR, and a higher proportion of crescents than the others groups, which are indicators of nephritis [28]. Patients with MIDD had higher levels of albumin and $\mathrm{SCr}$, more global sclerosis and interstitial fibrosis, and lower eGFRs, which might have led to worse prognosis [29]. Patients with PGNMID had the lowest paraprotein detection rate, which is consistent with the findings of a previous study [30], although the difference was not statistically significant. In the PGNMID group, $\mathrm{SCr}$ was the lowest and these patients had low serum C3 levels, which are indicators of complement pathway activation and better prognosis with immune suppressive treatment [31].
Most of our patients underwent special treatment including chemotherapy, and immunosuppressive therapy. Chemotherapy was more widely administered in the MM group than in the MGRS group, and patients with MM exhibited more partial renal response, but no major response. While $\mathrm{SCr}, \mathrm{Hb}$, serum $\kappa / \lambda$ ratio were independent risk factors for ESRD in the MGRS group, no independent risk factors were found in the MM group. The overall mortality rate of MM and MGRS was low due to active chemotherapy and the clone-directed approach [32, 33]. In the MGRS group, immunosuppressive therapy was only administered in patients with Cryo-GN and PGNMID, because they had complement activated proliferative glomerulonephritis, and no detectable clone in serum was found in patients with PGNMID. These patients were empirically prescribed with immunosuppressive medications and were relatively responsive, similar to the findings of previous reports [34-37]. Patients with MGUS and renal monoIg only received immunosuppressive therapy and after 4 years of follow-up on average, most patients achieved a major response and none of them died. Burwick et al. [38] reported that the progression of renal malfunction in these patients with MGUS to ESRD was $27.6 \%$ with severely reduced eGFR versus $4.0 \%$ with preserved eGFR. However, only one (5.3\%) patient progressed to ESRD in the MGUS group in our study, with slightly reduced eGFR. The overall survival was also reasonably high in the MGUS group, which was similar to that in the MGRS and MM groups, but renal survival was relatively low in the MM group. The Kaplan-Meier analysis revealed better renal and overall survival in the MGUS and renal monoIg groups than in the MGRS and MM groups, indicating that organ damage associated with MIg resulted in significantly worse outcomes. Additionally, patients with MIDD exhibited significantly poorer renal survival than patients with MGRS indicating that early diagnosis and aggressive therapy are especially important for these patients.

The limitations of this study were its retrospective design and the lack of long-term follow-up. Additionally, we were unable to analyze abnormal free light chain due to limited detection in a few patients, and complete hematological data could not be analyzed during follow-up.

\section{Conclusion}

The clinicopathological changes in patients with MGRS were between those in patients with MM and MGUS. The treatment of both MGRS and MM was more intensive, and the overall mortality rate was low. Both MGUS and renal monoIg alone exhibited fewer clinicopathological characteristics than MGRS and MM, and the treatment was mostly focused on primary renal diseases. 


\section{Abbreviations}

Mlg: Monoclonal immunoglobulin; MGUS: Monoclonal immunoglobulinemia of unknown significance; MGRS: Monoclonal gammopathy of renal significance; MM: Multiple myeloma; Renal monolg: Monotypic immunoglobulin in renal tissue; IgAN: IgA nephropathy; IMN: Idiopathic membranous nephropathy; SLE: Systemic lupus erythematosus; IF: Immunofluorescence; $\mathrm{Hb}$ : Hemoglobin; SCr: Serum creatinine; sIFE/UIFE: Serum/urine immunofixation electrophoresis; IFTA: Interstitial fibrosis/tubular atrophy; ESRD: End-stage renal disease; SD: Standard deviation; ANOVA: Analysis of variance; LCDD: Light chain deposit disease; LN: Lupus nephritis; Cryo-GN: Cryoglobulinemic glomerulonephritis; PGNMID: Proliferative glomerulonephritis with monoclonal IgG deposits; MIDD: Monoclonal immunoglobulin deposition disease; C3GN: C3 glomerulonephritis; ITG: Immunotactoid glomerulonephritis; IS: Immunosuppression therapy; pl: Isoelectric point; HBV-GN: Hepatitis B-related glomerulonephritis.

\section{Acknowledgements}

We thank Pro. Yunqin Hu, who kindly provided a diagnosis support, and Tian Ye as well, who kindly provided a technical support.

\section{Authors' contributions}

XT contributed to the study design, fund support and paper draft; FW contributed to the fund and data analyses; JY and XL performed the pathological analyses and technical support; RY and BZ provided supervision. All authors reviewed the manuscript. All authors read and approved the final manuscript.

\section{Funding}

This work was supported by grants from the project of Zhejiang Medical and Health Plan [2020KY750, 2021 KY924]; and the project of National Natural Science Foundation of China [81803911].

\section{Availability of data and materials}

The datasets used or analyzed during the current study are available from the corresponding author on reasonable request.

\section{Declarations}

\section{Ethics approval and consent to participate}

All procedures performed in studies involving human participants were in accordance with the ethical standards of the Institutional Review Board of Hangzhou TCM Hospital Affiliated to Zhejiang Chinese Medical University and with the 1964 Helsinki Declaration and its later amendments or comparable ethical standards [2020KY036]. As this was a retrospective observational study with full patient anonymity, the requirement for written informed consent from patients was waived.

\section{Consent for publication}

Written informed consent for publication was waived from all participants.

\section{Competing interests}

The authors declare that there are no competing interests.

Received: 17 March 2021 Accepted: 22 June 2021

Published online: 03 July 2021

\section{References}

1. Kyle RA, Larson DR, Therneau TM, Dispenzieri A, Kumar S, Cerhan JR, Rajkumar SV. Long-term follow-up of monoclonal gammopathy of undetermined significance. N Engl J Med. 2018;378(3):241-9.

2. Kyle RA, Therneau TM, Rajkumar SV, Larson DR, Plevak MF, Offord $J R$, Dispenzieri A, Katzmann JA, Melton $\sqcup$ 3rd. Prevalence of monoclonal gammopathy of undetermined significance. N Engl J Med. 2006;354(13):1362-9.

3. Kyle RA, Durie BG, Rajkumar SV, Landgren O, Blade J, Merlini G, Kröger $\mathrm{N}$, Einsele $\mathrm{H}$, Vesole DH, Dimopoulos M, et al. Monoclonal gammopathy of undetermined significance (MGUS) and smoldering (asymptomatic) multiple myeloma: IMWG consensus perspectives risk factors for progression and guidelines for monitoring and management. Leukemia. 2010;24(6):1121-7.

4. Kyle RA, Therneau TM, Rajkumar SV, Offord JR, Larson DR, Plevak MF, Melton $\sqcup$ 3rd. A long-term study of prognosis in monoclonal gammopathy of undetermined significance. N Engl J Med. 2002;346(8):564-9.

5. Rajkumar SV, Kyle RA, Therneau TM, Melton LJ 3rd, Bradwell AR, Clark RJ, Larson DR, Plevak MF, Dispenzieri A, Katzmann JA. Serum free light chain ratio is an independent risk factor for progression in monoclonal gammopathy of undetermined significance. Blood. 2005;106(3):812-7.

6. Klomjit N, Leung N, Fervenza F, Sethi S, Zand L. Rate and predictors of finding monoclonal gammopathy of renal significance (MGRS) lesions on kidney biopsy in patients with monoclonal gammopathy. J Am Soc Nephrol. 2020;31(10):2400-11.

7. Stamatova D, Theilmann L, Spiegelberg C. A hepatoid carcinoma of the pancreatic head. Surg Case Rep. 2016;2(1):78.

8. Steiner N, Göbel G, Suchecki P, Prokop W, Neuwirt H, Gunsilius E. Monoclonal gammopathy of renal significance (MGRS) increases the risk for progression to multiple myeloma: an observational study of 2935 MGUS patients. Oncotarget. 2018;9(2):2344-56.

9. Katafuchi R, Nagae H, Masutani K, Nakano T, Mitsuiki K. Clinicopathological significance of light chain deposition in IgA nephropathy. Clin Exp Nephrol. 2021;25:621.

10. Vignon M, Cohen C, Faguer S, Noel LH, Guilbeau C, Rabant M, Higgins S, Hummel A, Hertig A, Francois $\mathrm{H}$, et al. The clinicopathologic characteristics of kidney diseases related to monotypic IgA deposits. Kidney Int. 2017;91(3):720-8.

11. Nagae H, Tsuchimoto A, Tsuruya K, Kawahara S, Shimomura Y, Noguchi H, Masutani K, Katafuchi R, Kitazono T. Clinicopathological significance of monoclonal IgA deposition in patients with IgA nephropathy. Clin Exp Nephrol. 2017;21(2):266-74

12. Leung N, Bridoux F, Batuman V, Chaidos A, Cockwell P, D'Agati VD, Dispenzieri A, Fervenza FC, Fermand JP, Gibbs S, et al. The evaluation of monoclonal gammopathy of renal significance: a consensus report of the International Kidney and Monoclonal Gammopathy Research Group. Nat Rev Nephrol. 2019;15(1):45-59.

13. Sethi S, Rajkumar SV, D'Agati VD. The complexity and heterogeneity of monoclonal immunoglobulin-associated renal diseases. J Am Soc Nephrol. 2018;29(7):1810-23.

14. Gertz MA, Comenzo R, Falk RH, Fermand JP, Hazenberg BP, Hawkins PN, Merlini G, Moreau P, Ronco P, Sanchorawala V, et al. Definition of organ involvement and treatment response in immunoglobulin light chain amyloidosis (AL): a consensus opinion from the 10th International Symposium on Amyloid and Amyloidosis, Tours, France, 18-22 April 2004. Am J Hematol. 2005;79(4):319-28.

15. Sicard A, Karras A, Goujon JM, Sirac C, Bender S, Labatut D, Callard P, Sarkozy C, Essig M, Vanhille P, et al. Light chain deposition disease without glomerular proteinuria: a diagnostic challenge for the nephrologist. Nephrol Dial Transpl. 2014;29(10):1894-902.

16. Li XM, Rui HC, Liang DD, Xu F, Liang SS, Zhu XD, Huang XH, Liu ZH, Zeng $\mathrm{CH}$. Clinicopathological characteristics and outcomes of light chain deposition disease: an analysis of 48 patients in a single Chinese center. Ann Hematol. 2016;95(6):901-9.

17. Chauvet $S$, Bridoux $F$, Ecotière $L$, Javaugue $V$, Sirac $C$, Arnulf $B$, Thierry A, Quellard N, Milin S, Bender S, et al. Kidney diseases associated with monoclonal immunoglobulin M-secreting B-cell lymphoproliferative disorders: a case series of 35 patients. Am J Kidney Dis. 2015;66(5):756-67.

18. Ciocchini M, Arbelbide J, Musso CG. Monoclonal gammopathy of renal significance (MGRS): the characteristics and significance of a new metaentity. Int Urol Nephrol. 2017;49(12):2171-5.

19. Liang D, Liu J, Liang S, Xu F, Cheng Z, Huang X, Zeng C, Liu Z. Types of $M$ protein and clinicopathological profiles in patients with monoclonal gammopathy of renal significance. J Nephrol. 2020. https://doi.org/10. 1007/s40620-020-00817-X.

20. Yu XJ, Zhang X, Li DY, Wang SX, Zhou FD, Zhao MH. Renal pathologic spectrum and clinical outcome of monoclonal gammopathy of renal significance: a large retrospective case series study from a single institute in China. Nephrology. 2020;25(3):202-11.

21. Heher EC, Rennke HG, Laubach JP, Richardson PG. Kidney disease and multiple myeloma. Clin J Am Soc Nephrol. 2013;8(11):2007-17.

22. Boumediene A, Oblet C, Oruc Z, Duchez S, Morelle W, Huynh A, Pourrat J, Aldigier JC, Cogné M. Gammopathy with IgA mesangial deposition 
provides a monoclonal model of IgA nephritogenicity and offers new insights into its molecular mechanisms. Nephrol Dial Transpl. 2011;26(12):3930-7.

23. Al-Hussain T, Hussein MH, Al Mana H, Akhtar M. Renal involvement in monoclonal gammopathy. Adv Anat Pathol. 2015;22(2):121-34.

24. Motwani SS, Herlitz L, Monga D, Jhaveri KD, Lam AQ. Paraprotein-related kidney disease: glomerular diseases associated with paraproteinemias. Clin J Am Soc Nephrol. 2016;1 1(12):2260-72.

25. Nasr SH, Fidler ME, Cornell LD, Leung N, Cosio FG, Sheikh SS, Amir AA, Vrana JA, Theis JD, Dogan A, et al. Immunotactoid glomerulopathy: clinicopathologic and proteomic study. Nephrol Dial Transpl. 2012;27(11):4137-46.

26. Ravindran A, Fervenza FC, Smith RJH, Sethi S. C3 glomerulopathy associated with monoclonal Ig is a distinct subtype. Kidney Int. 2018;94(1):178-86.

27. Kourelis TV, Kumar SK, Gertz MA, Lacy MQ, Buadi FK, Hayman SR, Zeldenrust S, Leung N, Kyle RA, Russell S, et al. Coexistent multiple myeloma or increased bone marrow plasma cells define equally high-risk populations in patients with immunoglobulin light chain amyloidosis. J Clin Oncol. 2013;31(34):4319-24

28. Ramos-Casals M, Stone JH, Cid MC, Bosch X. The cryoglobulinaemias. Lancet. 2012;379(9813):348-60.

29. Nasr SH, Valeri AM, Cornell LD, Fidler ME, Sethi S, D'Agati VD, Leung N. Renal monoclonal immunoglobulin deposition disease: a report of 64 patients from a single institution. Clin J Am Soc Nephrol. 2012;7(2):231-9.

30. Bhutani G, Nasr SH, Said SM, Sethi S, Fervenza FC, Morice WG, Kurtin PJ, Buadi FK, Dingli D, Dispenzieri A, et al. Hematologic characteristics of proliferative glomerulonephritides with nonorganized monoclonal immunoglobulin deposits. Mayo Clin Proc. 2015;90(5):587-96.

31. Nasr SH, Satoskar A, Markowitz GS, Valeri AM, Appel GB, Stokes MB, Nadasdy T, D'Agati VD. Proliferative glomerulonephritis with monoclonal lgG deposits. J Am Soc Nephrol. 2009;20(9):2055-64.

32. Blimark CH, Turesson I, Genell A, Ahlberg L, Björkstrand B, Carlson K, Forsberg K, Juliusson G, Linder O, Mellqvist UH, et al. Outcome and survival of myeloma patients diagnosed 2008-2015. Real-world data on 4904 patients from the Swedish Myeloma Registry. Haematologica. 2018;103(3):506-13.

33. Finkel KW, Cohen EP, Shirali A, Abudayyeh A. Paraprotein-related kidney disease: evaluation and treatment of myeloma cast nephropathy. Clin J Am Soc Nephrol. 2016;1 1(12):2273-9.

34. Roccatello D, Baldovino S, Rossi D, Mansouri M, Naretto C, Gennaro M, Cavallo R, Alpa M, Costanzo P, Giachino O, et al. Long-term effects of antiCD20 monoclonal antibody treatment of cryoglobulinaemic glomerulonephritis. Nephrol Dial Transplant. 2004;19(12):3054-61.

35. Rossi D, Sciascia S, Fenoglio R, Ferro M, Baldovino S, Kamgaing J, Ventrella F, Kalikatzaros I, Viziello L, Solfietti L, et al. Cryoglobulinemic glomerulonephritis: clinical presentation and histological features, diagnostic pitfalls and controversies in the management. State of the art and the experience on a large monocentric cohort treated with B cell depletion therapy. Minerva Med. 2021;112(2):162-74

36. Gumber R, Cohen JB, Palmer MB, Kobrin SM, VogI DT, Wasserstein AG, Nasta SD, Bleicher MB, Bloom RD, Dember L, et al. A clone-directed approach may improve diagnosis and treatment of proliferative glomerulonephritis with monoclonal immunoglobulin deposits. Kidney Int. 2018;94(1):199-205

37. Guiard E, Karras A, Plaisier E, van DuongHuyen JP, Fakhouri F, Rougier JP, Noel LH, Callard P, Delahousse M, Ronco P. Patterns of noncryoglobulinemic glomerulonephritis with monoclonal Ig deposits: correlation with lgG subclass and response to rituximab. Clin J Am Soc Nephrol. 2011;6(7):1609-16.

38. Burwick N, Adams SV, Todd-Stenberg JA, Burrows NR, Pavkov ME, O'Hare AM. Association of monoclonal gammopathy with progression to ESKD among US veterans. Clin J Am Soc Nephrol. 2018;13(12):1810-5.

\section{Publisher's Note}

Springer Nature remains neutral with regard to jurisdictional claims in published maps and institutional affiliations.
Ready to submit your research? Choose BMC and benefit from:

- fast, convenient online submission

- thorough peer review by experienced researchers in your field

- rapid publication on acceptance

- support for research data, including large and complex data types

- gold Open Access which fosters wider collaboration and increased citations

- maximum visibility for your research: over $100 \mathrm{M}$ website views per year

At BMC, research is always in progress.

Learn more biomedcentral.com/submissions 